\title{
UWB Channel Modeling Improvement in Indoor Line-of-Sight (LOS) Environments
}

\author{
Vahid Tabataba Vakili, Dariush Abbasi-Moghadam, Mostafa Yahyaabadi \\ School of Electrical Engineering, Department of Telecommunications, Iran University of Science \& Technology (IUST), \\ Narmak, Iran \\ E-mail:Abbasi@ee.iust.ac.ir,Vakily@iust.ac.ir,yahyaabadi.mostafa@gmail.com \\ Received August 1, 2010; revised September 19, 2010; accepted October 21, 2010
}

\begin{abstract}
Channel measurement and modeling are important issues when designing ultra wideband (UWB) communication systems. A Precise model of the channel response is inevitable for designing a UWB telecommunication system. In this article signal propagation in indoor environment and LOS condition is evaluated and the appropriate model of this scenario is presented. Parameters such as the power delay profile, mean excess delay, delay spread, "NP${ }_{10 \mathrm{~dB}}$ " are analyzed and simulated. Based on the analysis results, the proposed model is presented. This model is based on Two-cluster approach but its average power delay profile is described with power function and cluster time of the arrival is modeled by the modified exponential distribution. Finally UWB channel parameters of the proposed model, Saleh and Valenzuela (S-V) and Two-cluster models are compared. Measurement and simulation results show that considerable improvement for mean excess delay, delay spread and " $\mathrm{NP}_{10 \mathrm{~dB}}$ " of proposed model comparing with $\mathrm{S}-\mathrm{V}$ and Two-cluster models, this means the channel is better described, which mean the channel is described more precisely.
\end{abstract}

Keywords: Channel Modeling, S-V Model, Power Delay Profile, Ultra Wideband

\section{Introduction}

UWB technology has been employed for several decades in military and commercial communications applications like high-speed mobile local area networks, imaging and surveillance systems, ground penetration radars, automotive sensors, medical monitors and recently wireless personal area networks. FCC has allocated band width from $3.1 \mathrm{GHz}$ to $10.6 \mathrm{GHz}$ to ultra wideband systems [1]. In recent years, these systems have gained more attention because of their advantages over narrowband systems. This system's RF signal consists of ultra short pulses with low power spectral density. Low transmission power $(-41.3 \mathrm{dBm})$ and large bandwidth together render the power spectral density of the transmitted signal extremely low, which allows the frequency-overlay of a UWB system with other existing radio systems such as GPS, IEEE802.11x and WLNA [2]. Spread-spectrum communication systems using ultra-short impulses have seen a renewed interest because of its fine resolution in delay to the order of several nanoseconds though at the cost of an ultra wide frequency band.

Channel transmission is a propagation environment through which the signal passes from transmitter to receiver. The propagation channel influences design aspects such as construction of the matched filter, choice of the Rake receiver structure, and search algorithms for geolocation of transceivers. As propagation environment of ultra wideband is usually indoor and crowded, the transmitted signal passes through different paths to receiver like other wireless channels, so the received signal is a combination of multi-path components which has a destructive form over the transmitted signal. These different multipath components are realized by different delays, various phases and amplitudes, therefore these three parameters should be included in the channel model. Precise modeling of channel is essential for designing UWB systems. S-V model which has been considered as the accepted standard by IEEE802.15.3a committee is the most well known model for modeling ultra wideband channels [3]. But S-V model is a standard model for wireless propagation in NLOS and is not precise for LOS. Also one of the other problems of this model is differentiation of clusters and statistical modeling of them [4]. For solving these issues, another model has been offered which is called Two-cluster [5]. This 
model is based on S-V model for ultra wideband channel including several stochastic clusters while in this model only two deterministic clusters are considered. Unfortunately, since in Two-cluster model, the second cluster is larger that S-V model clusters, we cannot define the average power delay profile with exponential function. A model has been proposed in this paper in which the average power delay profile is defined by power function.

The paper is organized as follows: in Section 2, measurement conditions are explained, in Section 3, Twocluster and S-V models are introduced, Section 4 presents the proposed model, while in Section 5 new model is simulated and finally concluding remarks are presented in Section 6.

\section{Measurement}

Propagation measurements have been made to characterize the UWB signal propagation channel. Using short pulse with sub-nanosecond width, the impulse response of the channel can be observed. Figure $\mathbf{1}$ is the block diagram of the experimental setup. Transmitter consists of a pulser, a periodic pulse generator, and a transmitting antenna. The periodic pulse generator generates a regular frame clock signal with period of 1 microsecond, which triggers the pulser. The periodic pulse generator is also connected to a DSO (Digitized Sampling Scope) by coaxial cable to provide a trigger signal for a measurement of the receiving antenna output. The pulser generates Gaussian-shaped pulses with sub-nanosecond duration. Figure 2 shows the output signals of a typical pulser. Since the antenna system differentiates and filters the pulser's output, a more complex waveform is detected by the DSO. Incoming signal is differentiated at receiving antenna and observed at DSO. The DSO takes samples over many periods of the transmission to construct one received waveform, and averages several such waveform measurements. Figure 2(b) shows a typical signal measured by the DSO in an indoor setting. The LOS path component of the signal is shown in the first two or three nanoseconds of the response, and is followed by a number of multipath components.

Experiments were carried out in a laboratory with size

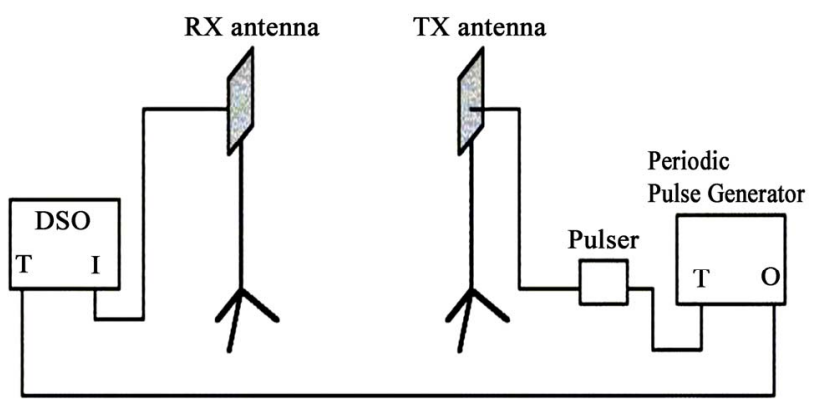

Figure 1. A block diagram of the measurement apparatus [6]. of $2.7 \mathrm{~m}$ (height), $13.5 \mathrm{~m}$ (length) and $8.5 \mathrm{~m}$ (wide). The transmitter antenna has been located $165 \mathrm{~cm}$ far from the floor near the southern wall. LOS receiver, which is named F1, has been located 9.5 meter far from the transmitter, near the western wall. The distance from receiver antenna to floor is $120 \mathrm{~cm}$. In Figure 3, the plan of laboratory has been shown from above. In receiver location, a square matrix of $7 \times 7=49$ sensors with 15 cm spacing has been provided.

CLEAN algorithm was used to extract the CIR from measurements data of the received waveforms [8,9]. The power delay profile $[10,11]$, defined by (1) is averaged over 49 measured channels and is plotted in Figure 4.

$$
P D P=\frac{1}{M} \sum_{m}^{M=49}\left|h_{m}(\tau)\right|^{2}
$$

Experimental data have been used as criteria for examination of model's accuracy. Therefore, each model impulse response is a random process which cloud be adequately presented by three random variables (parameters): mean excess delay, RMS delay spread $\mathrm{NP}_{10 \mathrm{~dB}}$. $\mathrm{CDF}$ and mean of each parameters are computed by simulation using at least 1000 runs. It is obvious, CDF and mean of a model which is closer to measured CDF and mean will be selected as a more precise channel model as described in the following section.
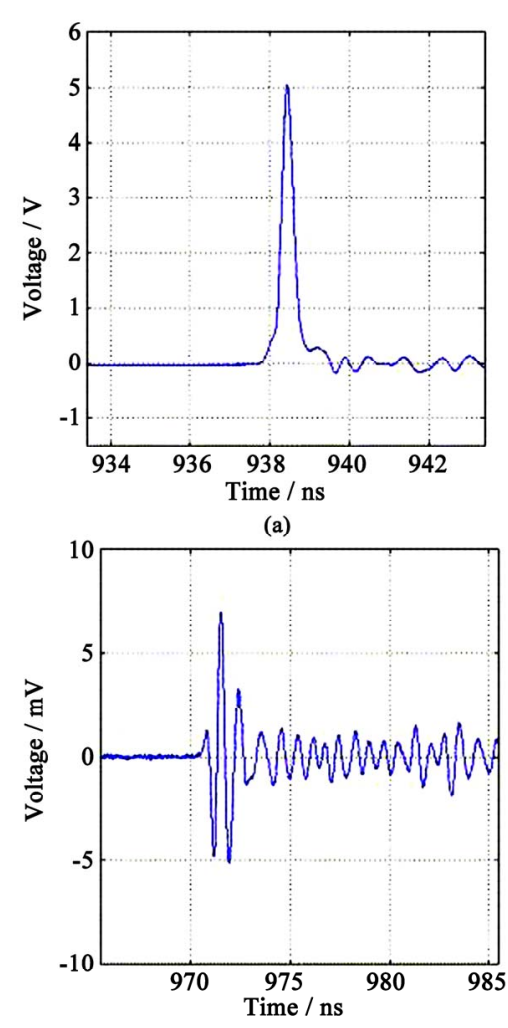

(b)

Figure 2. (a) Pulser output; (b) Received signal. 


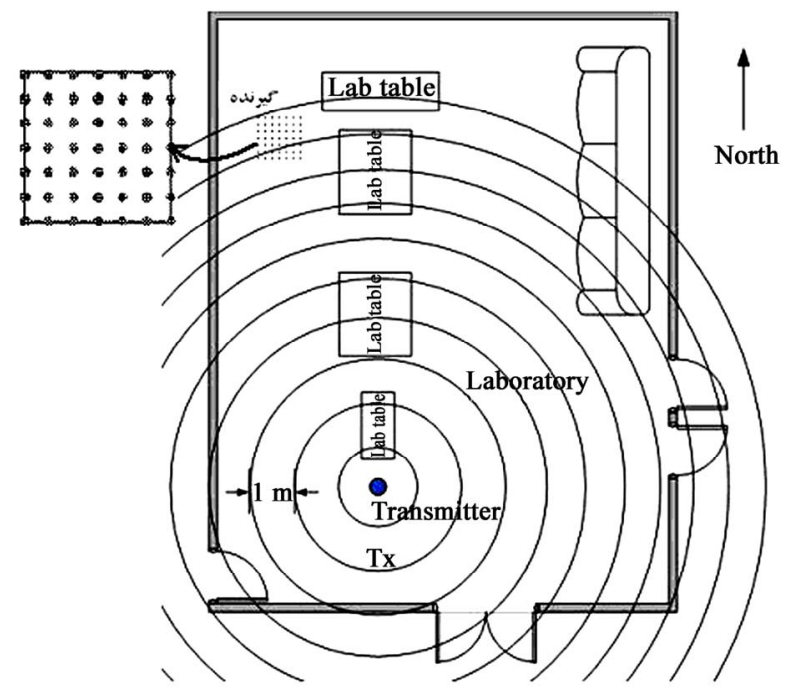

Figure 3. Plan of laboratory where the LOS propagation measured experiment was performed [7].

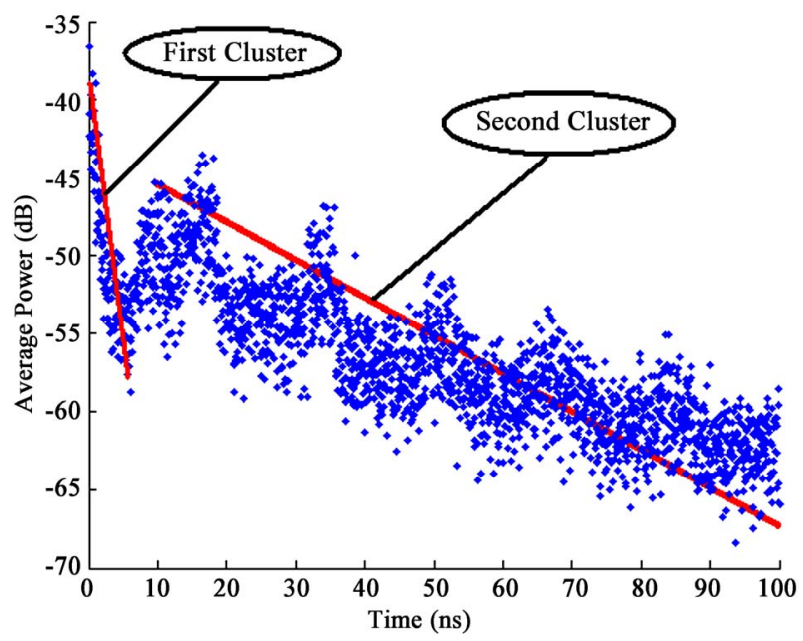

Figure 4. Average power delay profile versus excess delay in a semi logarithmic scale for the 49 LOS locations in the laboratory.

\section{S-V and Two-Cluster Model}

It can be seen from Figure 4, due to multipath components in UWB systems, each cluster consists of several rays. This definition of ultra wideband systems were introduced by "Saleh and Valenzuela" for the first time [3, 11-13]. The following impulse response has been proposed for UWB channel [14]:

$$
h(t)=X \sum_{l=0}^{L} \sum_{k=0}^{K} \alpha_{k, l} \delta\left(t-T_{l}-\tau_{k, l}\right)
$$

where $L$ denotes the number of clusters, $l$ is the cluster index, $k$ is ray index, $L$ is total number of clusters, $K$ is the total number of rays in each cluster. $X$ Indicates shad- owing factor and $\alpha_{k, l}$ is ray gain for $\mathrm{k}^{\text {th }}$ ray of $\mathrm{l}^{\text {th }}$ cluster. These two parameters are modeled by lognormal distribution. Also $T_{l}$ is $\mathrm{l}^{\text {th }}$ cluster time arrival, $\tau_{k, l}$ is time arrival of $\mathrm{k}^{\text {th }}$ rays of $\mathrm{l}^{\text {th }}$ cluster. Assuming time of arrival distribution as Poisson, time interval distribution should be considered as exponential.

$\mathrm{S}-\mathrm{V}$ model has several problems. First, this model is not accurate for LOS and indoor applications. Second, finding the accurate values of parameters like cluster arrival rate and inter cluster exponential decay constant is difficult for modeling cluster behaviors based on experimental data .This would require some specific algorithms of distinguishing clusters from one another. In order to compensate for this disadvantage, an UWB channel model with two deterministic clusters and stochastic arriving rays within each cluster were proposed [5].This model is based on S-V model, but the only difference is that instead of several clusters with random time of arrival, only two clusters are considered with deterministic time of arrival (Figure 4). Also, instead of determining the gain of first ray of each cluster stochastically in S-V model, the gain of first ray in first and second clusters are computed deterministically. The other model components, i.e., gain and time of arrival of next rays in each two cluster are modeled statistically. The channel has the following response:

$$
h(t)=\sum_{k=0}^{M-1} \alpha_{k, 0} \delta\left(t-\tau_{k, 0}\right)+\sum_{k=0}^{N-1} \alpha_{k, 1} \delta\left(t-T_{m}-\tau_{k, 1}\right)
$$

where $\alpha_{k, l}$ is multipath gain coefficient, $T_{m}$ is the time interval between two clusters, $\tau_{\mathrm{k}, 0}$ is the delay of the kth multipath component relative to the first cluster arrival time, $\tau_{k, l}$ is the time delay of the $k$ th multipath component relative to the second cluster arrival time $\left(T_{m}\right)$, $M$ is the number of paths in the first cluster, and $N$ is the number of paths in the second cluster. The parameters of this model can be divided into deterministic and stochastic parts. In order to calculate the deterministic parameters, it is enough to have length, width and height of the room, electromagnetic properties of reflecting surface, polarization and bandwidth of transmitted and received signal. The statistical parameters are modeled like S-V model.

\section{Proposed Model}

The proposed model is based on Two-cluster model. The difference is that in this model, the average power delay profile is defined by power function instead of exponential function and the time interval between times of rays' arrival is modeled by modified exponential function instead of exponential model. The reasons for above modifications are explained in following sections. 


\subsection{Deterministic Part of Model}

Deterministic part of the model includes the path gain of the first ray of two clusters and their time delay. In this model, LOS ray is considered as the first ray of the first cluster. The arrival time of this ray is assumed to be zero and its gain is determined based on path loss characteristic of free space:

$$
\alpha_{0,0}=\frac{\lambda}{4 \pi d}, \lambda=\frac{c}{\sqrt{f_{m}}}
$$

where $c$ is light speed, $f_{m}$ is the geometrical mean of the upper and lower signal frequency limits, $d$ is the distance between the transmitter and receivers' antenna. The first ray of the second cluster is a ray which is reflected once from six reflecting surfaces (four walls, ceiling and floor of laboratory). The arrival time of this ray is calculated as follows:

$$
T_{m}=\frac{\min \left\{L_{i}\right\}-L_{0}}{C}, i=1,2,3,4,5,6
$$

where $L_{0}$ is the length of direct path and $L_{i}$ is the length of reflected rays. For computing the gain of this ray except path loss of free space, we should consider the loss resulting from reflection [15]. Therefore:

$$
\begin{aligned}
\alpha_{0,1}= & \frac{L_{0}}{L_{0}+\min \left\{L_{i}\right\}} \\
& \times\left\{ \pm K\left[1+\frac{2 k}{1-k^{2}} \exp \left[(1+K) a T_{m}\right]\right]\right\}, i=1 \ldots 6
\end{aligned}
$$

With $\mathrm{K}=(1-\mathrm{k}) /(1+\mathrm{k})$, and $k=\frac{\sqrt{\varepsilon_{r}-\cos ^{2} \varphi}}{\varepsilon_{r}-\sin \varphi}$ for vertical polarization and $k=\frac{\sin \varphi}{\sqrt{\varepsilon_{r}-\cos ^{2} \varphi}}$ for horizontal polarization and $a=120 \pi \sigma c / \varepsilon_{r}$. Here, $\varepsilon_{r}$ and $\sigma$ are relative dielectric constant and the conductivity of reflecting surface [16] respectively. By the abovementioned definitions, the deterministic parameters are calculated and presented in Table 1.

\subsection{Statistical Part of Model}

The gain and arrival time of the next rays in both clusters are modeled statistically. The gain of these rays is modeled as follows, but with slight difference with S-V model:

$$
\alpha_{k, 0 / 1}=P_{k, 0 / 1} \beta_{k, 0 / 1}, k \geq 1
$$

where $P_{k, 0 / 1}$ will be \pm 1 with equal probability. $\beta_{k, 0 / 1}$ is lognormal fading of rays with standard deviation of $\delta$ :

$$
20 \log \left(\beta_{k, 0 / 1}\right) \propto \operatorname{Normal}\left(\mu_{k, 0 / 1}, \delta^{2}\right), k \geq 1
$$

Determination of $\mu_{k, 0 / 1}$ depends on the functionali ty of average power delay profile. In Two-cluster $\mathrm{m}$ odel, the average power delay profile is defined by exponential function:

$$
E\left[\beta_{k, 0 / 1}^{2}\right]=\alpha_{0,0 / 1}^{2} \cdot e^{-\frac{\tau_{k, 0 / 1}}{\gamma_{0 / 1}}} \quad, k \geq 1
$$

As Figure 5 shows, the average power delay profile does not follow the exponential function accurately (the continuous line shows the exponential function). The reason why is the fact that the second large cluster consists of several small clusters are not considered in modeling of the average PDP as exponential function of time.

In order to achieve more accuracy, we define the average power delay profile as a power function of time:

$$
E\left[\beta_{k, 0 / 1}^{2}\right]=\left(\alpha_{0,0 / 1}\right)^{2}\left(\frac{\tau_{k, 0 / 1}+T_{m}}{T_{m}}\right)^{-\omega}
$$

where $\alpha_{k, l}$ is ray gain for multi-path, $\tau_{k, l}$ is arrival time of $\mathrm{k}^{\text {thl }}$ rays of $\mathrm{l}^{\text {th }}$ cluster and $T_{m}$ is the second cluster arrival time.

The adjustment of power delay profile with power function is shown in Figure 5. The dashed line shows power function. As Figure 5 shows, the average power

Table 1. Values of deterministic parameters of model.

\begin{tabular}{cccc}
\hline & Excess Delay (ns) & \multicolumn{2}{c}{ Path gain } \\
\cline { 3 - 4 } & 0 & $\begin{array}{c}\text { Vertical } \\
\text { Polarization }\end{array}$ & $\begin{array}{c}\text { Horizontal } \\
\text { Polarization }\end{array}$ \\
\hline LOS & 5.44 & 0.009 & 0.009 \\
Reflected & & 0.0058 & 0.0069 \\
\hline
\end{tabular}

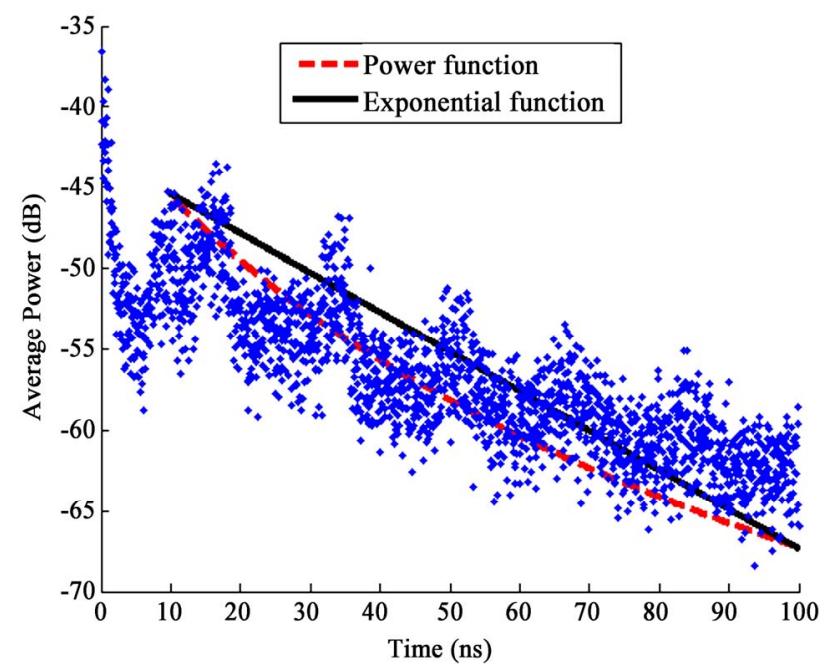

Figure 5. PDP modeled with Power function and Exponential function. 
delay profile based on power function, compared with exponential, is more fitted to measured PDP data. The mean and variance of error between averaged PDP based on power function and measured PDP and also error between averaged PDP based on exponential function and measured PDP are presented in Table 2. As this table shows the difference between variances of these two function models is $2.0166 \mathrm{~dB}$ respectively. Hence we should compute $\mu_{k, l}$ based on new definition of average power delay profile.

Since $\beta_{k, 0 / 1}$ is modeled with lognormal distribution (Equation 8), we can have:

$$
\left|\beta_{k, 0 / 1}\right|^{2}=e^{\frac{\ln (10)}{10} \cdot \operatorname{Normal}\left(\mu_{k, 0 / 1}, \delta^{2}\right)}
$$

Hence:

$$
\begin{gathered}
B \propto \operatorname{Normal}\left(m_{b}, \delta_{b}^{2}\right), z=e^{B} \Rightarrow E\left[z^{2}\right]=e^{2\left(m_{b}+\delta_{b}^{2}\right)} \\
k \times \operatorname{Normal}\left(m, \delta^{2}\right)=\operatorname{Normal}\left(k m, k^{2} \delta^{2}\right)
\end{gathered}
$$

From (12) and (13) we have:

$$
\begin{aligned}
& E\left[\beta_{k, 0 / 1}^{2}\right]=e^{2(m+\text { Var })}, \\
& m=\frac{\ln (10) \cdot \mu_{k, 0 / 1}}{20}, \operatorname{Var}=\frac{(\ln (10))^{2} \delta^{2}}{(20)^{2}}
\end{aligned}
$$

By equalizing (10) and (14) we conclude that:

$$
\mu_{k, l}=\frac{20 \ln \alpha_{0,0 / 1}-\omega 10 \ln \left(\frac{\tau_{k, 0 / 1}+T_{m}}{T_{m}}\right)}{\ln (10)}-\frac{\delta^{2} \ln (10)}{20}
$$

Therefore, in the proposed model $\beta_{\mathrm{k}, 0 / 1}$ is the lognormal fading term with standard deviation $\sigma$ and $\mu_{k, l}$ which was defined in (Equation 15). Arrival time of ray in Two-cluster model described with exponential function, but in proposed model, arrival time of rays described with modified exponential function (Equation 16). Comparison of arrival time of ray of these two models with measured data shows that the proposed model is improved. So in the proposed model, the time interval between times of rays' arrival is modeled with modified exponential distribution:

$$
\begin{aligned}
P\left(\tau_{k, 0 / 1} \mid \tau_{k-1,0 / 1}\right)= & (1-b) \lambda_{1} \times \exp \left(-\lambda_{1}\left(\tau_{k, 0 / 1}-\tau_{k-1,0 / 1}\right)\right) \\
& +\lambda_{2} b \times \exp \left(-\lambda_{2}\left(\tau_{k, 0 / 1}-\tau_{k-1,0 / 1}\right)\right)
\end{aligned}
$$

where $b$ is close to 1 . The values of $\lambda, \gamma, \delta, b$, which were used in simulation have been shown in Table 3 .

\section{Simulation Results}

In this section simulation result of Two-cluster model, $\mathrm{S}-\mathrm{V}$ model and the proposed model have been compared. This comparison has been done regarding mean and cu- mulative distribution function of three key parameters, i.e., mean excess delay, RMS delay spread and " $\mathrm{NP}_{10 \mathrm{~dB}}$ ". First, several channels have been simulated for both previous models and proposed model by MATLAB 7 Then the parameters of each simulated channel are computed and recorded and the mean and cumulative distribution function for each parameter was obtained from these recorded values. In order to compare and evaluate these models, the obtained values of mean and cumulative distribution function in measured data have been considered as reference. In Table 4 the result of mean value of parameters is compared. The average value of mean excess delay in the proposed model, Two-cluster model and S-V model are 14.68 ns, 15.28 ns and 16.04 ns, respectively. Also, the mean value for RMS delay spread in the proposed model, Two-cluster model and S-V model are 17.54 ns 17.62 ns and 18.76 ns, respectively. The mean value of " $\mathrm{NP}_{10 \mathrm{~dB}}$ " in the proposed model, Two-cluster model and S-V model are 7.85, 8.91 and 8.93, respectively and " $\mathrm{NP}_{10 \mathrm{~dB}}$ " in measured data is 7.51. Compare these values with obtained values from measurements show that the average relative error proposed model parameters, mean excess delay and delay spread and " $\mathrm{NP}_{10 \mathrm{~dB}}$ " in comparison with the Two-cluster model were 4.2 percent, 0.45 percent, 14.11 percent, compared S-V model, respectively, 9.5 percent, 6.88 percent and 14.38 percent decrease.

Table 2. Variances of error.

\begin{tabular}{ccc}
\hline & Exponential function & Power function \\
\hline error variance & 24.2897 & 22.2731 \\
\hline
\end{tabular}

Table 3. Value of parameters used in proposed model simulation.

\begin{tabular}{cc}
\hline$\lambda_{1}(1 / \mathrm{ns})$ & $\mathbf{0 . 1 5}$ \\
\hline$\lambda_{2}(1 / \mathrm{ns})$ & 1.54 \\
$\mathbf{b}$ & $\mathbf{0 . 0 9 5}$ \\
$\gamma(\mathrm{ns})$ & 17.6 \\
$\delta(\mathrm{dB})$ & 3.3941 \\
\hline
\end{tabular}

Table 4. Average value of key parameter.

\begin{tabular}{ccccc}
\hline & Measurement & $\begin{array}{c}\text { S-V } \\
\text { model }\end{array}$ & Two-clusters & $\begin{array}{c}\text { Proposed } \\
\text { model }\end{array}$ \\
\hline $\begin{array}{c}\text { Mean Excess } \\
\text { Delay(ns) }\end{array}$ & 14.25 & 16.04 & 15.28 & 14.68 \\
$\begin{array}{c}\text { RMS Delay } \\
\text { Spread (ns) } \\
\text { NP }_{\text {10dB }}\end{array}$ & 17.52 & 18.76 & 17.62 & 17.54 \\
\hline
\end{tabular}




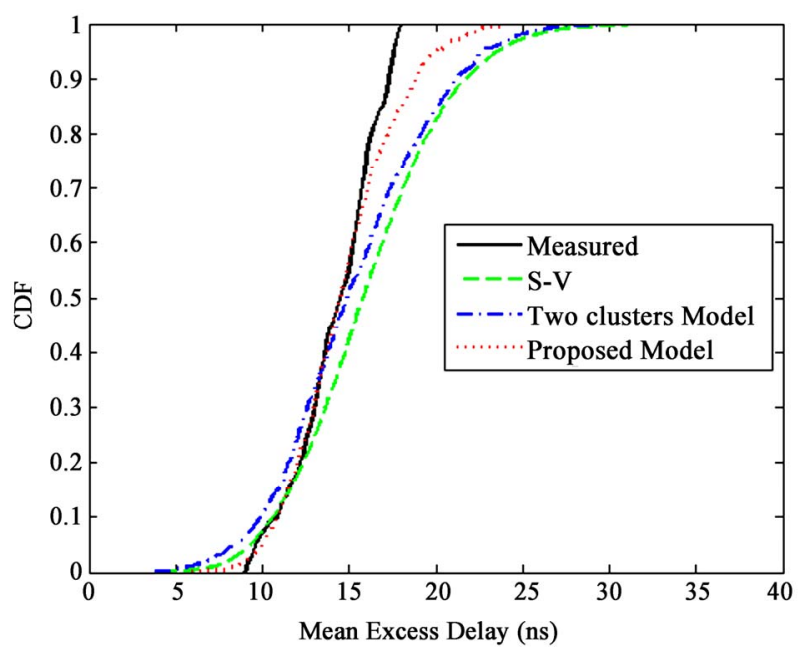

Figure 6. CDF of mean excess delay.

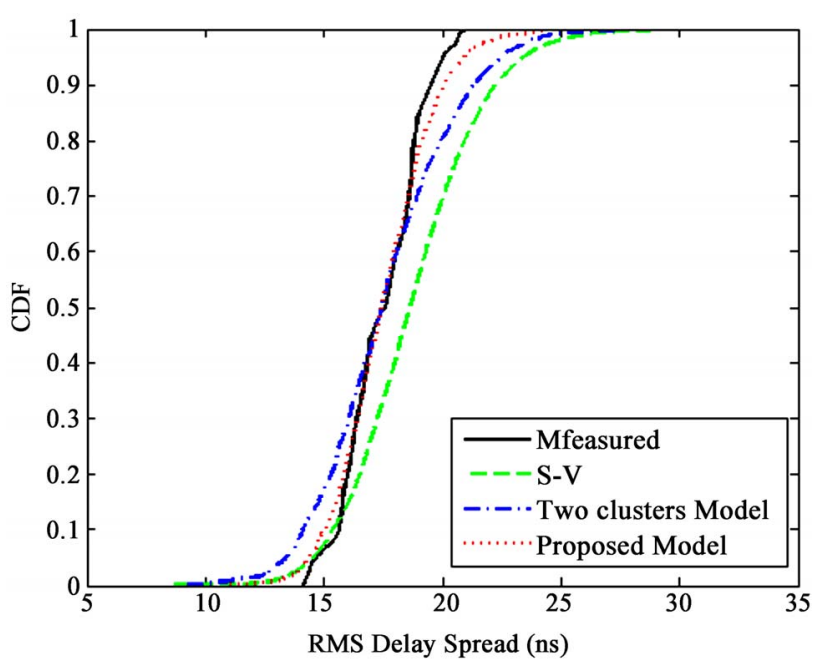

Figure 7. CDF of RMS delay spread.

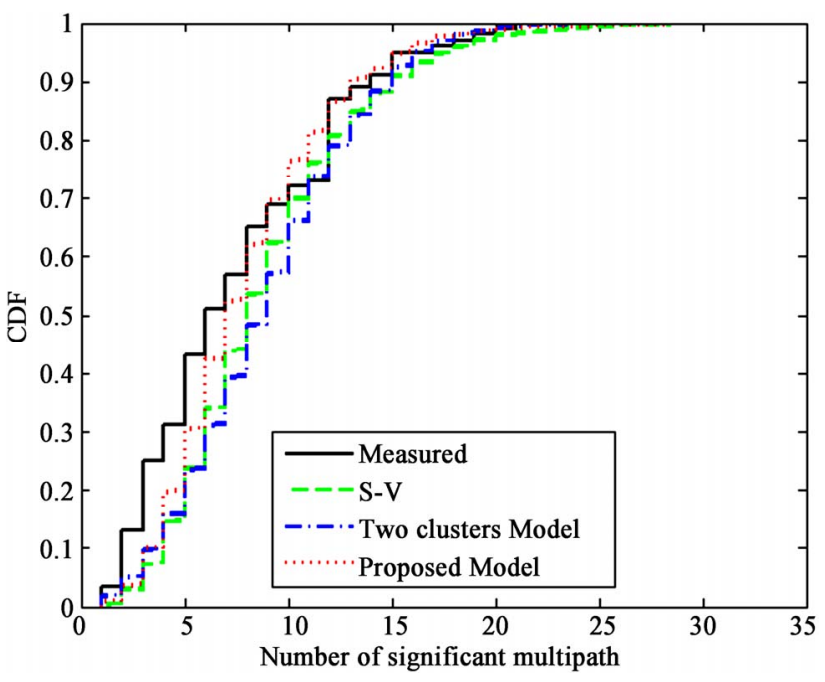

Figure 8. CDF of number of significant multipaths.
In Figures 6-8 the CDF of mean excess delay, RMS delay spread and " $\mathrm{NP}_{10 \mathrm{~dB}}$ " have been drawn for three models. As it can be seen, CDF of mean excess delay and RMS delay spread in the proposed model is closer to the measured values than Two-cluster model and S-V model. Also CDF of " $\mathrm{NP}_{10 \mathrm{~dB}}$ " is better for the proposed model comparing to the previous models. The ray arrival time in the proposed model, which is described with exponential distribution using $\lambda_{1}, \lambda_{2}$, is more precise compared to S-V and Two-cluster models. Furthermore, the ray arrival time and the ray gain of the proposed model are closer to the experimental results.

\section{Conclusion}

In this paper, a model has been presented for indoor LOS UWB channel. The proposed model for indoor LOS UWB channel doesn't need the description of clusters and knowing the mean parameters of arrival rate of cluster and their variance comparing to S-V model. Also, instead of approximating the gain of first ray with a mean value, it is determined accurately and substituted in the model. Moreover instead of simulating the time arrival of several clusters with statistical distribution, we should compute deterministically and use the time arrival of two clusters. As in Two-cluster model, several clusters of S-V model are considered as the second cluster. In proposed model, the average power delay profile was approximated with power function. Furthermore in the proposed model, times were modeled by the modified exponential function. Results show that the cumulative distribution functions of three parameters of proposed model has been recovered comparing with $\mathrm{S}-\mathrm{V}$ model and Two-cluster model. The mean value for mean excess delay and RMS delay spread and " $\mathrm{NP}_{10 \mathrm{~dB}}$ " in the proposed model have been recovered $0.6 \mathrm{~ns}, 0.08 \mathrm{~ns}$ and 1.06 comparing with Two-cluster model and $1.36 \mathrm{~ns}$, 1.22 ns and 1.08 comparing with S-V model. Therefore the proposed model fits better for data and has less variance, hence it can model the channel better than S-V and Two-cluster model in LOS environment.

\section{References}

[1] M. Z. Win and R. A. Scholtz, "Characterization of Ultra-Wide Band Width Wireless Indoor Communication Channel: A Communication Theoretical View," IEEE JSAC, Vol. 20, No. 9, December 2002, pp. 1613-1627.

[2] Multiband OFDM Physical Layer Proposal for IEEE 802.15 Task Group 3a. http://www.ieee802.org/15/

[3] A. Saleh and R. Valenzuela, "A Statistical Model for Indoor Multipath Propagation,” IEEE Journal on Selected Areas in Communications, Vol. 5, No. 2, February 1987, pp. 128-137. 
[4] S. Venkatesh and J. Ibrahim, "A New 2-Cluster Model for Indoor UWB Channel Measurement," IEEE in Antenna and Propagation Society Symposium, Vol. 1, June 2004, pp. 946-949.

[5] Y. Wang, N. T. Zhang, Q. Y. Zhang and Z. Z. Zhang, "Characterizing Ultra-Wide Band Indoor Line-of-Sight Wireless Channel,” Elsevier Journal of Systems Engineering and Electronics, Vol. 18, No. 4, December 2007, pp. 673-678.

[6] http://ultra.usc.edu/uwb_database/

[7] D. Cassioli, M. Z. Win and A. F. Molisch, "The Ultra-Wide Bandwidth indoor Channel: From Statistical Model to Simulations," IEEE Journal on Selected Areas in Communications, Vol. 20, No. 6, August 2002, pp. 1247-1257.

[8] Y. Wang and N. T. Zhang, "A New Multi-Template CLEAN Algorithm for UWB Channel Impulse Response Characterization,” IEEE Conference on Communication Technology, Guilin, November 2006, pp. 1-4.

[9] C. Buccella, M. Feliziani and G. Manzi, "Detection and Localization of Defects in Shielded Cables by Time-Domain Measurement with UWB Pulse Injection and Clean Algorithm Post Processing," IEEE Transactions on Electromagnetic Compatibility, Vol. 46, No. 4, November 2004, pp. 597-605.
[10] J. H. Kim and Y. K. Yoon, "The Multipath Delay Spread Model for the LOS Case," Antennas and Propagation Society International Symposium, San Diego, July 2008, pp. 1-4.

[11] R. G. Vaughan and N. L. Scott, "Super-Resolution of Pulsed Multipath Channels for Delay Spread Characterization," IEEE Transactions on Communications, March 1995, Vol. 47, No. 3, pp. 343-347.

[12] Y. F. Chen, "Statistical Properties of IEEE UWB Channel Models and Their Application," IEEE 19th International Symposium on Indoor and Mobile Radio Communications, Cannes, September 2008, pp. 1-5.

[13] J. Wout, D. Jeffrey, V. Leen and M. Luc. "Statistical Analysis of the Power Delay Profile," Elsevier International Journal of Communications, In Press, Available online, 7 July 2009.

[14] J. Foerster, "Channel Modeling Sub-Committee Report Final,” IEEE P802.15-02/368r5-SG3a, 2002.

[15] V. Marshall and G. Skitek, "Electromagnetic Concept and Applications,” Prentice-Hall International, Inc., 1990.

[16] P. R. Barnes and F. M. Tesche, "On the Direct Calculation of Transient Plane Wave Reflected from a Finitely Conducting Half Space," IEEE Transaction on Electromagnetic Compatibility, Vol. 33, No. 2, May 1991, pp. 90-96. 